\title{
Keine Angst vor Fencheltee
}

\author{
Reinhard Saller
}

Zürich, Schweiz

Teezubereitungen aus Fenchelfrüchten (Foeniculi amari fructus, Foeniculi dulcis fructus) spielen seit dem Altertum in traditionellen und auch modernen Medizinsystemen (von China und Indien bis nach Europa) sowie in den verschiedenen Volksmedizinen eine grosse therapeutische Rolle [1]. Gerade im letzten Jahrzehnt hat neben phytochemischen Untersuchungen diesbezüglich die Anzahl ethnomedizinischer Studien erheblich zugenommen [1]. Der derzeitige Gebrauch umfasst unter anderem die innerliche Verwendung bei stillenden Müttern (z.B. Förderung der Milchsekretion) sowie bei Appetitlosigkeit, Bauchschmerzen, Blähungen, Verdauungsstörungen (Dyspepsie bei Kindern und Erwachsenen), Durchfall bei Säuglingen, Magen- und Darmkrämpfen, Husten und Bronchialkatarrh - ebenso zur Förderung der Menstruation und zur Linderung von Menstruationskrämpfen $[1,2]$. Äusserlich wird Fencheltee bei ekzematösen Erkrankungen des äusseren Auges (Blepharitis, Konjunktivitis) sowie als Augenwasser bei müden Augen und funktionellen Sehstörungen angewendet, ausserdem als Spülungen bei Rachenentzündungen [1].

Eine besondere Stellung besitzt Fencheltee als Spasmolytikum und Karminativum bei Säuglingen und Kleinkindern, da er neben seiner Wirksamkeit und hohen Akzeptanz als sehr gut verträglich gilt. Insgesamt zählt Fencheltee zu den am häufigsten verwendeten pflanzlichen Arznei-, Heil- und Hausmitteln bei Säuglingen und/oder Kleinkindern mit Bauchkrämpfen, Bauchschmerzen und Blähungen sowie bei Schwangeren und Stillenden [2,3]. Darüber hinaus wird er gerne als Getränk bei Säuglingen und Kleinkindern mit einem erhöhten Flüssigkeitsbedarf verabreicht.

Fenchelfrüchte gehören zu den estragolhaltigen Drogen [2-4]. Nach einer aktuellen umfangreichen Untersuchung variieren die Estragolmengen stark - je nach Handelsware zwischen 0,15 und 13,3 mg/g Droge (Trockenmaterial). In Teezubereitungen liegen die Konzentrationen wesentlich niedriger, d.h. zwischen 0,4 und 133,4 $\mu \mathrm{g} / 25 \mathrm{ml}$ Tee (zubereitet aus $1 \mathrm{~g}$ fein geschnittener Droge), entsprechend einer Extraktionsrate zwischen $<0,1$ und 2,5\% [4]. Diese Extraktionsrate liegt mindestens um eine Zehnerpotenz niedriger als die früher häufig angenommene Rate von 25-30\%. Es scheinen keine Unterschiede zwischen Bitter- oder Süssfenchel zu bestehen [4]. Mittlerweile bestätigen auch direkte Estragolbestimmungen in der Milch stillender Mütter, die fenchelhaltigen Tee tranken, ausserordentlich niedrige Konzentrationen (potenziell übertretende Substanzen liegen um etwa den Faktor 1000 niedriger als in den Ausgangsprodukten) [5]. Vergleichbar mit diesen Ergebnissen wurde auch keine geruchliche Beeinträchtigung der Muttermilch gefunden [5].

Durch die Risikoabschätzung und Mitteilung des deutschen Bundesinstituts für gesundheitlichen Verbraucherschutz und Veterinärmedizin (BgVV), des heutigen Bundesamtes für Verbraucherschutz und Lebensmittelsicherheit (BVL), vom 11.05.2001, in der aus Gründen der Vorsorge zur Einschränkung der Verwendung von Fenchel als Tee geraten wird, wurde eine intensive Fachdiskussion und gleichzeitig eine erhebliche Verunsicherung weiter Konsumentenkreise ausgelöst (siehe [3]). An die Hersteller von estragol- und methyleugenolhaltigen Produkten ging die Auflage, diese Inhaltsstoffe weitestgehend $\mathrm{zu}$ minimieren. Das Amt stützte sich auf tierexperimentelle Daten, die als Hinweise auf ein genotoxisches und kanzerogenes Risiko des unter anderem in Fenchelfrüchten enthaltenen Inhaltsstoffs Estragol gewertet wurden. Tierexperimentelle Daten lassen sich jedoch nicht zwanglos auf den Menschen übertragen. Dementsprechend konnte das Risiko nicht quantifiziert werden. Zudem lagen bzw. liegen keine klinischen oder epidemiologischen Daten vor, die ein kanzerogenes Risiko in irgendeiner Weise bestätigen würden. Die fachliche Kritik fokussierte vor allem auf die Frage der Übertragbarkeit der aus Tierexperimenten gewonnenen Daten auf den Menschen sowie auf die hohen Dosen der eingesetzten Reinsubstanz (Estragol), die vielfach höher waren als die Mengen, denen der Konsument von estragolhaltigen Nahrungs- und Arzneimitteln tatsächlich ausgesetzt ist $[1,3]$. Des Weiteren zeigen Studien zum Metabolismus von Estragol, dass sich der Stoffwechsel von Nagetieren und Menschen zumindest in quantitativer Hinsicht erheblich unterscheidet $[1,3]$.

\section{KARGER}

Fax +497614520714

Information@Karger.com

www.karger.com
Prof. em. Dr. med. Reinhard Saller

Institut für komplementäre und integrative Medizin

UniversitätsSpital Zürich

Sonneggstrasse 6, 8006 Zürich, Schweiz

reinhard.saller@gmx.ch 
Ein weiterer, nach wie vor in der Forschung, Risikoeinschätzung und öffentlichen Diskussion häufig und erheblich unterschätzter Gesichtspunkt ist der pharmakologisch-toxikologische Unterschied zwischen Wirkstoffen, die als Monosubstanzen verabreicht werden, und den gleichen Stoffen, die Bestandteile natürlicher Vielstoffgemische sind $[1,3]$. Fencheltee enthält als phytotherapeutisches Vielstoffgemisch (phytochemische Matrix), das gesamthaft auf den Organismus einwirkt, beispielsweise eine ganze Reihe von antioxidativ wirkenden und auch anderweitigen Substanzen, die antimutagene, zytoprotektive, antikanzerogene, antitumorale und chemopräventive Eigenschaften besitzen [1-3, 6-8]. Zudem können eine Reihe der weiteren Inhaltsstoffe des Fencheltees nicht nur Wirkungen von Estragol, sondern auch dessen Metabolismus und Pharmakokinetik beeinflussen [6]. Insgesamt könnte auch dadurch ein mögliches kanzerogenes Risiko von Estragol erheblich herabgesetzt und möglicherweise sogar aufgehoben werden.

Im November 2014 erschien ein Draft (Revision 1) der European Medicines Agency («Public statement on the use of herbal medicinal products containing estragole»), in dem mit ausführlichen Begründungen auf die entsprechende Problematik eingegangen wird [9]. In ihm wird ausgeführt, dass derzeit z.B. für einen Erwachsenen mit $50 \mathrm{~kg}$ Körpergewicht eine tägliche Menge an Estragol von 0,5 mg/Person als akzeptabel angesehen wird (bioverfügbare Menge); für Kinder mit einem Körpergewicht von z.B. 20 kg könnte die akzeptable tägliche Aufnahme 0,2 mg/Person betragen (jeweils beispielhafte Abschätzungen angepasst an das Körpergewicht). Da auch die Einnahmedauer bedeutsam ist, beziehen sich die Einschätzungen auf eine Kurzzeitanwendung von maximal 14 Tagen. Die Überlegungen umfassen auch die tägliche Aufnahme von Estragol über die Nahrung, die auf 0,5-5 mg/Tag geschätzt wird. Unter der Annahme einer realitätsgerechten maximalen Extraktionsrate aus Nahrungsmitteln von 2,5\% [4] und einer maximalen Gesamtmenge von $5 \mathrm{mg} /$ Tag Estragol dürfte die realistische (bioverfügbare) Estragolmenge $0,125 \mathrm{mg} /$ Person/Tag betragen. Je nach tatsächlicher Extraktionsrate könnte sie z.B. auch deutlich niedriger liegen.

Die Abschätzungen beziehen sich auch auf besonders sensible Bevölkerungsgruppen wie schwangere und stillende Frauen [9]. Die derzeitige wissenschaftliche Datenlage und die darauf basierenden Folgerungen erlauben es meiner Ansicht nach, zumindest die oben angesprochene, jeweils zeitlich beschränkte Verwendung von Fencheltee auch während der Schwangerschaft und Stillperiode sowie in der Kindheit als akzeptabel und sinnvoll einzustufen.

\section{Disclosure Statement}

Keine Interessenkonflikte.

\section{Literatur}

1 Badgujar SB, Patel VV, Bandivdekar AH: Foeniculum vulgare Mill: a review of its botany, phytochemistry, pharmacology, contemporary application, and toxicology. Biomed Res Int 2014;2014:842674.

2 Iten F, Saller R: Fencheltee im Kindesalter. Nutzen-Risiko-Abschätzung. Pädiatrische Praxis 2007;69:517-530.

3 Iten F, Saller R: Fencheltee: Risikoabschätzung der phytogenen Monosubstanz Estragol im Vergleich zum natürlichen Vielstoffgemisch. Forsch Komplementärmed Klass Naturheilkd 2004;11:104-108.
4 Van den Berg SJPL, Alhusainy W, Restani P, Rietjens IMCM: Chemical analysis of estragole in fennel based teas and associated safety assessment using the Margin of Exposure (MOE) approach. Food Chem Toxicol 2014;65:147-154.

5 Denzer MY, Kirsch F, Buettner A: Are odorant constituents of herbal tea transferred into human milk? J Agric Food Chem 2015;63:104-111.

6 Gori L, Gallo E, Mascherini V, Mugelli A, Vannacci A, Firenzuoli F: Can estragole in fennel seed decoctions really be considered a danger for human health? A fennel safety update. Evid Based Complement Alternat Med 2012;2012: 860542.
7 Singh B, Kale RK: Chemomodulatory action of Foeniculum vulgare (Fennel) on skin and forestomach papillomagenesis, enzymes associated with xenobiotic metabolism and antioxidant status in murine model system. Food Chem Toxicol 2008;46:3842-3850.

8 Choi EM, Hwang JK: Anti-inflammatory, analgesic and antioxidant activities of the fruit of Foeniculum vulgare. Fitoterapia 2004;75:557565.

9 (HMPC) EMACoHMP: Public statement on the use of herbal medicinal products 4 containing estragole. EMA/HMPC/137212/2005, Rev 1, 2014. 